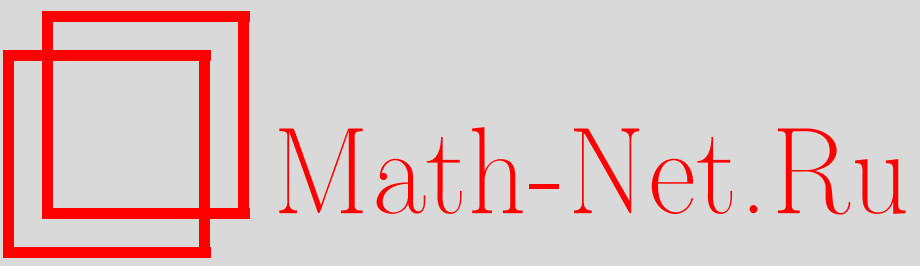

О. Костин, М. Крускал, Подвижные особые точки решений разностных уравнений, их связь с разрешимостью и исследование сверхстабильных фиксированных точек, ТМФ, 2002, том 133, номер 2, 160-169

DOI: https://doi.org/10.4213/tmf387

Использование Общероссийского математического портала Math-Net.Ru подразумевает, что вы прочитали и согласны с пользовательским соглашением

http://www.mathnet.ru/rus/agreement

Параметры загрузки:

IP: 34.229 .108 .108

26 апреля 2023 г., 17:52:28 
ТЕОРЕТИЧЕСКАЯ

И МАТЕМАТИЧЕСКАЯ

ФИЗИКА

Tом 133, № 2

ноябрь, 2002

(C) 2002 г.

О. Костин*, М. Крускал*

\title{
ПОДВИЖНЫЕ ОСОБЫЕ ТОЧКИ РЕШЕНИЙ РАЗНОСТНЫХ УРАВНЕНИЙ, ИХ СВЯЗЬ С РАЗРЕШИМОСТЬЮ И ИССЛЕДОВАНИЕ СВЕРХСТАБИЛЬНЫХ ФИКСИРОВАННЫХ ТОЧЕК
}

\begin{abstract}
Дается обзор приложений экспоненциальных асимптотик и теории анализируемых функций к разностным уравнениям при определении аналога свойства Пенлеве для них, а также набросок заключений относительно свойства разрешимости автономных уравнений первого порядка. Оказалось, что если выполнено свойство Пенлеве, то уравнения являются явно решаемыми; в противоположном случае, при дальнейших предположениях, интегралы движения порождают барьеры сингулярностей. Метод применяется к логистическому отображению $x_{n+1}=a x_{n}\left(1-x_{n}\right)$, для которого оказывается, что единственными случаями со свойством Пенлеве являются случаи, когда $a=-2,0,2$ и 4 , для которых явные решения действительно существуют; в противном случае ассоциированное сопряженное отображение порождает барьер сингулярностей.
\end{abstract}

Ключевые слова: суммирование по Борелю, экспоненциальные асимптотики, анализ особенностей, трансценденты Пенлеве.

\section{1. ВВЕДЕНИЕ}

Мы в общих чертах представляем новые методы для определения положения и типа особых точек некоторого вида решений разностных уравнений [1] и используем эту информацию для их анализа Пенлеве. Такой подход основывается на достижениях в исследованиях экспоненциальных асимптотик и теории анализируемых функций [2]-[6]. Обсудим сначала основные концепции.

Анализируемые функции, введенные Экаллем, являются в основном аналитическими функциями, которые в особых точках полностью описываются трансрядами почти так же, как аналитические функции в регулярных точках представляются сходяшимися рядами. В отличие от аналитических функций (не замкнутых относительно деления) и мероморфных функций (теряющих устойчивость относительно интегрирования и композиции), анализируемые функции предположительно являются замкнутыми относи-

*Department of Mathematics, Busch Campus-Hill Center Rutgers University, Piscataway, NJ, 08854, USA. E-mail: costin@math.rutgers.edu 
тельно всех операций, откуда следует общая картина этой теории, согласно которой все функции естественного происхождения являются анализируемыми. В частности, было показано, что решения многих классов дифференшиальных и разностных уравнений являются анализируемыми.

Трансряды, также введенные Экаллем, представляют собой “окончательное” обобщение рядов Тейлора. Трансряды являются формальными асимптотическими комбинациями степенных рядов, экспонент и логарифмов и содержат богатую информацию не только о локальном, но и о глобальном поведении функций. Одним из простейших нетривиальных примеров трансрядов является ряд

$$
\sum_{k=0}^{\infty} \frac{k !}{x^{k+1}}+C e^{-x}, \quad x \rightarrow+\infty
$$

Выражение (1.1) отличается от стандартных асимптотических разложений наличием за пределом всех порядков главного ряда еше и экспоненциально малого члена, который не охватывается классической асимптотичностью Пуанкаре. Более обшие трансряды, возникаюшие в обыкновенных дифференциальных и разностных уравнениях обшего типа, являются двусторонне-бесконечными рядами, члены которых даются степенями $x$, умноженными на экспоненты (см., например, (2.3)). Эти трансряды можно определить алгоритмически, и обычно они факториально расходятся, однако можно показать, что они суммируются по Борелю в некотором секторе в соответствуюшем смысле. В этом секторе функция, для которой трансряд суммируется, имеет хорошие аналитические свойства, а на краях сектора, как правило, появляются особые точки.

Соответствие между формальным миром и актуальными функциями, представляемое Экаллем как "окончательный” изоморфизм, осушествляемый обобшенным суммированием по Борелю, было строго установлено в ряде задач, включая обыкновенные дифференциальные уравнения, разностные уравнения и дифференциальные уравнения в частных производных.

\section{2. РАЗНОСТНЫЕ УРАВНЕНИЯ: СВОЙСТВО ИЗОЛИРОВАННОЙ ПОДВИЖНОЙ ОСОБОЙ ТОЧКИ}

Проблема интегрируемости была и остается предметом интенсивных исследований. В контексте дифференциальных уравнений сушествует множество эффективных критериев интегрируемости, среди которых решаюшую роль играет тест Пенлеве (многочисленные ссылки см., например, в [7]).

Определение аналога свойства Пенлеве для разностных уравнений оказывается более тонким.

Различные методы для исследования разрешимости были предложены Джоши [8], Абловицем с соавторами [9], Грамматикосом и Рамани (см. ссылки в [10]), Конте и Мюсеттом [11]; в работе [9] проводится также сравнительное обсуждение различных подходов. Ни один из них не является настоящим обобщением теста Пенлеве. Одна из трудностей состоит в том, как естественно и эффективно продолжить определенные на дис- 
кретном множестве решения $x(n)$ разностного уравнения на комплексную плоскость независимой переменной $n$. Вложение $x(n)$ должно быть выполнено с сохранением свойств. Для эффективности анализа важно, чтобы вложение $x(n) \sqsubset x(z)$ было естественным, конструктивным и единственным при подходящих условиях.

Конечно, решающее значение имеет то, что имеется бесконечно много значений функции $x(n)$; поскольку точкой сгущения $n$ является бесконечность, поведение $x$ на $\infty$ является ключевым. Поэтому вопрос сводится к тому, когда функцию определяют бесконечно много значений, а также к какому классу она принадлежит.

Чтобы проиллюстрировать это обстоятельство, начнем с достаточно тривиального примера. Предположим, что $x(n)$ выражается через сходяшиеся степенные ряды по $1 / n$ :

$$
x_{n}=\sum_{k=1}^{\infty} c_{k} n^{-k}
$$

Тогда естественно было бы определить $x(n) \sqsubset x(z)$ как

$$
x(z)=\sum_{k=1}^{\infty} c_{k} z^{-k}
$$

при достаточно больших $z$. Единственность обеспечивается аналитичностью $x(z)$ на бесконечности. По аналитичности вложение $\sqsubset$ сохраняет все свойства.

Мы не можем просто полагаться на аналитическое продолжение, поскольку имеется лишь несколько классов уравнений, решения которых даются сходящимися степенными рядами. Однако, как было обнаружено Экаллем и совсем недавно подробно доказано в обшем виде Брааксмой [3], широкий класс разностных уравнений произвольного порядка допускает формальные решения в виде суммируемых по Борелю трансрядов. Класс, рассмотренный Брааксмой, имеет вид

$$
\mathbf{x}_{n+1}=\mathbf{G}\left(\mathbf{x}_{n}, n\right)=\hat{\Lambda}\left(I+\frac{1}{n} \hat{A}\right) \mathbf{x}_{n}+\mathbf{g}\left(n, \mathbf{x}_{n}\right),
$$

где $\mathbf{G}$ аналитична на бесконечности по $n$ и в нуле по $x_{n}$ при допушении о том, что рассматривается случай общего вида [3]. В частности, накладывается нерезонансное условие

$$
\mu_{m}=\mathbf{k} \cdot \boldsymbol{\mu} \quad(\bmod 2 \pi i),
$$

где $\mathbf{k} \in \mathbb{N}^{n}$, если и только если $\mathbf{k}=\mathbf{e}_{m}$. Решения этих уравнений в виде трансрядов имеют много общего с решениями дифференциальных уравнений в виде трансрядов. При некоторых $m_{1}, 0 \leqslant m_{1} \leqslant n$, имеем

$$
\tilde{\mathbf{x}}(n)=\sum_{\mathbf{k} \in \mathbb{N}^{m} 1} \mathbf{C}^{\mathbf{k}} e^{-\mathbf{k} \cdot \boldsymbol{\mu} n} n^{\mathbf{k} \cdot \mathbf{a}} \tilde{\mathbf{y}}_{\mathbf{k}}(n) .
$$

Здесь $\boldsymbol{\mu}=\left(\mu_{1}, \ldots, \mu_{m_{1}}\right)$ и $\mathbf{a}=\left(a_{1}, \ldots, a_{m_{1}}\right)$ зависят только от рекурренции (они являются собственными значениями соответственно $\hat{\Lambda}$ и $\hat{A}), \mathbf{C}-$ свободный параметр, а $\tilde{\mathbf{y}}_{\mathbf{k}}$ 
формальные ряды по отрицательным целым степеням $n$, независимые от С. Число $m_{1}$ выбирается так, чтобы все экспоненты в (2.3) стремились к нулю в выбранном секторе.

Брааксма показал, что $\tilde{\mathbf{y}}_{\mathbf{k}}(n)$ являются равномерно суммируемыми по Борелю в $\mathbf{k}$. Пусть $\mathbf{Y}_{\mathbf{k}}=\mathcal{L}^{-1} \tilde{\mathbf{y}}_{\mathbf{k}}(n)$. Тогда $\mathbf{Y}_{\mathbf{k}}(p)$ аналитичны в окрестности $\mathbb{R}^{+}$(в действительности даже в большем секторе). Определив

$$
\mathbf{y}_{\mathbf{k}}=\int_{0}^{\infty} e^{-n p} \mathbf{Y}_{\mathbf{k}}(p) d p
$$

получаем равномерные оценки $\left|\mathbf{y}_{\mathbf{k}}\right|<A^{\mathbf{k}}$ и, следовательно, ряд

$$
\mathbf{x}(n)=\sum_{\mathbf{k} \in \mathbb{N}^{m_{1}}} \mathbf{C}^{\mathbf{k}} e^{-\mathbf{k} \cdot \boldsymbol{\mu} n} n^{\mathbf{k} \cdot \mathbf{a}} \mathbf{y}_{\mathbf{k}}(n)
$$

классически сходится при достаточно больших $n$. Брааксма показал, что $\mathbf{x}(n)$ является актуальным решением уравнения (2.1). Естественно заменить $n$ на $z$ в уравнении (2.4) и определить

$$
\mathbf{x}(z)=\sum_{\mathbf{k} \in \mathbb{N}^{m} 1} \mathbf{C}^{\mathbf{k}} e^{-\mathbf{k} \cdot \boldsymbol{\mu} z} z^{\mathbf{k} \cdot \mathbf{a}} \mathbf{y}_{\mathbf{k}}(z) .
$$

Если $z$ и все константы вешественны и $\mu_{i}<0$, то функции (2.6) представляют собой специальный случай анализируемых функций Экалля. Как было объяснено выше, мы допускаем комплексность $z$ и констант при условии, что $\operatorname{Re}(\mathbf{k} \cdot \boldsymbol{\mu} z)>0$.

Решаюшим является то, что значения $\mathbf{y}(n)$ для всех достаточно больших $n$ фиксируют разложение единственным образом. В работе [1] показано, что при подходяших условиях две различные анализируемые функиии не могут быть согласованными на множестве точек сәущения в бесконечности. Ниже приведен упрошенный вариант теоремы из работы [1].

TeOpema 1 ([1]). Пyсmb

$$
\left(\mathbf{Z} \cdot \boldsymbol{\mu}=0 \quad(\bmod 2 \pi i) \quad \text { npu } \quad \mathbf{Z} \in \mathbb{Z}^{p}\right) \Leftrightarrow \mathbf{Z}=0,
$$

а $\mathbf{x}(z)$ задается уравнением (2.6). Если $\mathbf{x}(n)=0$ для всех достаточно больиих $n \in \mathbb{N}$, то функция $\mathbf{x}(z)$ тожсдественно равна нулю.

Анализируемые функции ведут себя во многих отношениях как аналитические. В частности, в качестве важных свойств мы имеем принцип сохранения соотношений и единственности продолжения с множеств с точками сгушения. В сделанных в работе [3] предположениях, обеспечивающих суммируемость по Борелю, введем следующее определение.

ОПРЕДЕЛЕНИЕ 1. Если $\mathbf{x}(n)$ задается представлением (2.5), сходяшимся при больших $n$, то функция $\mathbf{x}(z)$, задаваемая при больших z уравнением (2.6), называется анализируемым вложением функции $\mathbf{x}(n)$ из $\mathbb{N}$ в сектор в $\mathbb{C}$.

Теперь, имея подходящую процедуру аналитического продолжения, можно определить свойство изолированной подвижной особой точки (СИПОТ) как естественное обобщение свойства Пенлеве для разностных уравнений. Аналогично случаю дифференциальных уравнений, потребуем, чтобы все решения были свободны от "плохих" подвижных особых точек. 
ОПРЕДЕлЕниЕ 2. Разностное уравнение имеет СИПОТ, если все подвижные особые точки всех его решений являются изолированными.

ЗАМЕЧАНИЕ 1. Мы используем обычное соглашение о том, что изолированные особые точки исключают точки ветвления, кластеры полюсов и барьеры сингулярностей.

ЗАмЕчАНИЕ 2. Для определения особых точек трансасимптотические методы сшивки, введенные для дифференциальных уравнений в работе [5], можно с небольшими изменениями распространить на разностные уравнения.

\section{3. КЛАССИФИКАЦИЯ НЕКОТОРЫХ РАЗНОСТНЫХ УРАВНЕНИЙ С СИПОТ. РАЗРЕШИМОСТЬ}

Рассмотрим автономные разностные уравнения вида $x_{n+1}=G\left(x_{n}\right)$, где $G$ мероморфна и имеет притягиваюшие неподвижные точки. Более общий анализ дается в работе [1] Запишем

$$
x_{n+1}=G\left(x_{n}\right):=a x_{n}+F\left(x_{n}\right)
$$

и ограничимся для простоты случаем, когда $F(0)=F^{\prime}(0)=0$ и $0<|a|<1$. Имеется однопараметрическое семейство решений, представленных как сходящиеся при достаточно больших $n$ простые трансряды, вида

$$
x_{n}=x_{n}(C)=\sum_{k=1}^{\infty} e^{n k \ln a} C^{k} D_{k}
$$

для заданных значений $D_{k}$, независимых от $C$. Анализируемое вложение $x$ (см. определение 1) имеет вид

$$
x(z)=x(z ; C)=\sum_{k=1}^{\infty} e^{z k \ln a} C^{k} D_{k}
$$

и является аналитическим при достаточно больших z. Для того чтобы найти СИПОТ, рассмотрим свойства $x(z)$ при значениях $z$, лежащих вне области сходимости $(3.3)$, и особые точки $x(z)$.

ЗАмЕчаниЕ 3 . Поскольку уравнение (3.1) является нелинейным, то, несмотря на то что (3.3) имеет один непрерывный параметр, решений может быть больше. Этот вопрос рассмотрен в работе [1].

3.1. Вложение и свойство отображения сопряжения. По теореме Пуанкаре о сопряжении, примененной к $x_{n+1}=G\left(x_{n}\right)$, сушествует единственное отображение $\phi$ со свойствами

$$
\phi(0)=0, \quad \phi^{\prime}(0)=1, \quad \phi \text { аналитично в } 0,
$$

такое что из $x_{n}=\phi\left(X_{n}\right)$ следует $X_{n+1}=a X_{n}$. Отображение $\phi$ является отображением сопряжения для $x_{n+1}$ с линеаризацией $X_{n+1}$. Имеем $X_{n}=a^{n} X_{0}=C a^{n}$.

Получим продолжение $x$ из $\mathbb{N}$ в $\mathbb{C}$ посредством

$$
x_{n}=\phi\left(C a^{n}\right) \sqsubset x(z):=\phi\left(C a^{z}\right) .
$$


Тогда отображение сопряжения удовлетворяет уравнению

$$
\phi(a z)=G(z)=a \phi(z)+F(\phi(z)) .
$$

Как и ожидалось, в силу единственности вложения в определении 1 имеет место следующая

ЛЕмма 1. При заданнъх предположсениях для уравнений типа (3.1) вложсения с помощью анализируемости и с помощью отображсения сопряжсения (3.5) согласуются. Решения $x(z ; C)$ имеют только изолированные подвижные особые точки, если и только если ф имеет только изолированные особые точки.

СлЕДСТВИЕ. Необходимое условие того, чтобъ (3.1) имело СИПОТ, состоит в том, чтобъ отображение сопряжения ф вокруг каждой устойчивой неподвижной точки функиии $G$ и тех ее итераций, которые являются мероморфными (ви$\partial а G^{[m]}=\underbrace{G \circ \cdots \circ G}_{m})$, аназ исключением изолированных особъх точек.

3.2. Автономные уравнения с СИПОТ. В работе [1] мы классифицировали уравнения вида (3.1) по отношению к СИПОТ. Аналогично случаю обыкновенных дифференциальных уравнений первого порядка, только уравнения Риккати имеют это свойство. С другой стороны, эти уравнения являются явно разрешимыми, и следовательно, автономные уравнения первого порядка, имеюшие СИПОТ, можно решить явно.

ТЕОРема 2. Уравнение (3.1) при заданных предположсениях не обладает СИПОТ за исключением тех случаев, когда для некоторого $с \in \mathbb{C}$

$$
G(z)=\frac{a z}{1+c z}
$$

В этом случае $1 / x_{n}=a^{-n}(C-c /(a-1))+c /(a-1)$.

Интересно также, что в случае отсутствия СИПОТ аналитические свойства решений препятствуют сушествованию хороших констант движения. Это обсуждается в следуюшем разделе.

3.3. Случай, когда СИПОТ не имеет места. Проиллюстрируем эту ситуацию, когда $G$ - полином. Неожиданный вывод состоит в том, что для многочлена $G$ без СИПОТ константы движения (определяемые как функции $C(x, n)$, постоянные вдоль траекторий) развивают барьеры сингулярностей вдоль некоторых фрактальных замкнутых кривых $\partial \mathcal{K}_{p}$ (см. ниже). В окрестности начала координат функция $\phi$ обратима, и таким образом при малых $x$ мы получаем из $x_{n}=\phi\left(a^{n} C\right)$, что $C=a^{-n} Q\left(x_{n}\right)$, где $Q=\phi^{-1}$.

На рисунке (построенном с помошью С и Maple) приведены множества Джулиа $\partial \mathcal{K}_{p}$ простого отображения для $G=a x(1-x)$ при $a=0.5, a=0.9$ и $a=1$, соответственно. Множество $\mathcal{K}_{p}$ представляет собой внутренность этих кривых. В этом случае $\mathcal{K}_{p}$ состоит из начальных условий, при которых решение итерации сходится к нулю. Множество 

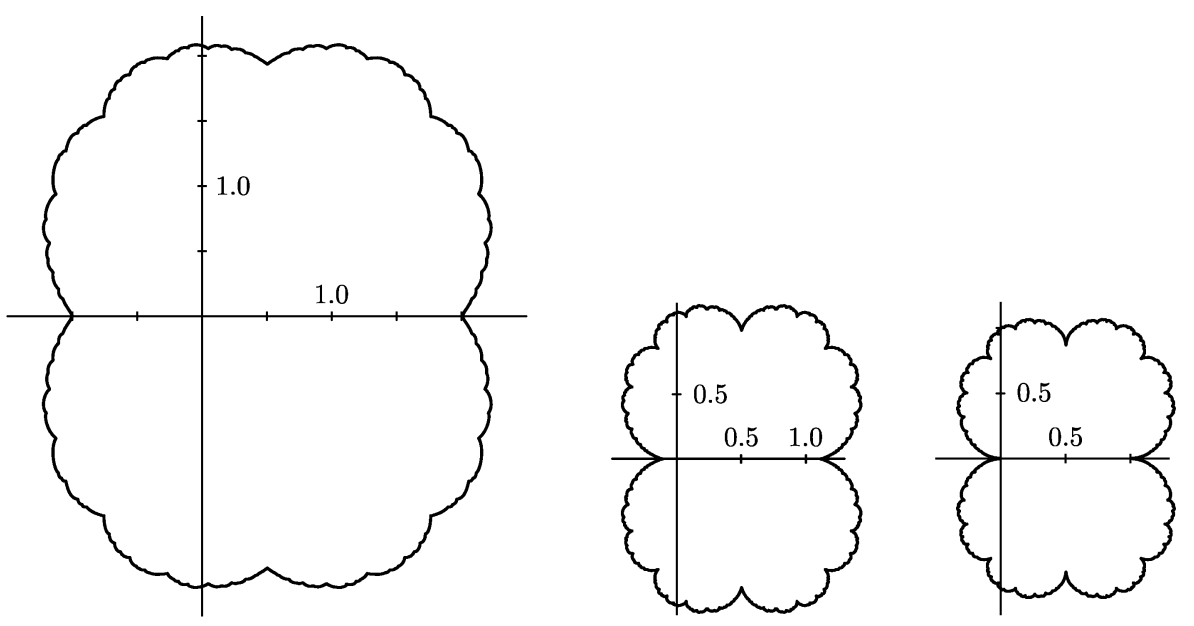

Джулиа является замкнутой кривой нетривиальной фрактальной размерности. Исчерпьвающее обсуж дение множеств Джулиа и итераций рациональных отображений см. в работе [2].

ТЕОРЕма 3. Предположим, что $G$ является полиномиальным отображсением с притягивающей неподвижной точкой в начале координат. Обозначим через $\mathcal{K}_{p}$ (см. рисунок) максимальную связную компоненту начала координат в множест-

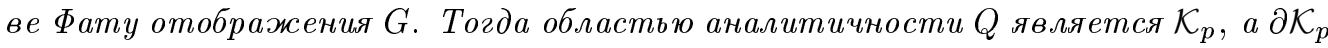
является барьером сингулярностей для $Q$.

Доказательства этих результатов можно найти в работе [1]. Логистическое отображение, которое достаточно подробно обсуждается в следующем разделе, дает очень простую иллюстрацию ряда существенных явлений.

\section{4. АНАЛИЗ ЛОГИСТИЧЕСКОГО ОТОБРАЖЕНИЯ В СУПЕРУСТОЙЧИВОЙ НЕПОДВИЖНОЙ ТОЧКЕ. БЕСКОНЕЧНОСТЬ}

Покажем, что уравнение $x_{n+1}=a x_{n}\left(1-x_{n}\right)$ имеет СИПОТ, если и только если $a \in\{-2,0,2,4\}$. Случай $a=0$ не требует анализа. С другой стороны, беря $y=1 / x$, получаем

$$
y_{n+1}=\frac{y_{n}^{2}}{a\left(y_{n}-1\right)} .
$$

При малых $y_{0}$ уравнение (4.1) в старшем порядке есть $a y_{n+1}=-y_{n}^{2}$ с решением $-y_{0}^{2^{n}} a^{-2^{n}-1}$. Удобно искать решения уравнения (4.1) в виде $y_{n}=F\left(y_{0}^{2^{n}} a^{-2^{n}}\right)$. Начальное условие влечет $F(0)=0, F^{\prime}(0)=-a$. Обозначая $y_{0}^{2^{n}} a^{-2^{n}}=z$, получаем, что функциональное соотношение, которому удовлетворяет $F$, имеет вид

$$
F\left(z^{2}\right)=\frac{F(z)^{2}}{a(F(z)-1)} .
$$


Лемма 2. Существует единственная аналитическая функция $F$ в окрестности начала координат, удовлетворяющая (4.2) и такая, что $F(0)=0$ и $F^{\prime}(0)=-а$. Эта функиия имеет только изолированные особые точки в $\mathbb{C}$ тогда и только тогда, когда $a \in\{-2,2,4\}$. В последнем случае уравнения (4.1) и (4.2) могут бъть решены явно (см. (4.5), (4.8) и (4.9)).

Если $а \notin\{-2,2,4\}$, то единичный диск является барьером сингулярностей функиии F. Интеграл движения $Q=F^{-1}$ аналитичен в нуле и, если $а \notin(-2,2,4)$, то $\partial \mathcal{K}_{p}-$ барвер сингулярностей $Q$.

ДокАЗАТЕльство. Запишем

$$
F(z)=\frac{a}{2} F\left(z^{2}\right)-\frac{1}{2} \sqrt{a^{2} F\left(z^{2}\right)^{2}-4 a F\left(z^{2}\right)}
$$

(при этом мы выбираем ветвь в соответствии с $F(0)=0, F^{\prime}(0)=-a$ ) и возьмем $F(z)=$ $-a z+h(z)$. Это приводит к уравнению для $h$ :

$$
h(z)=a z+\frac{a}{2}\left(h\left(z^{2}\right)-a z^{2}\right)-\frac{1}{2} \sqrt{4 a^{2} z^{2}-4 a h\left(z^{2}\right)+a^{2}\left(h\left(z^{2}\right)-a z^{2}\right)^{2}}=\mathcal{N}(h) .
$$

Можно непосредственно показать, что при малых $\epsilon$ функция $\mathcal{N}$ сжимающая в шаре радиуса $|a|^{2}$ в пространстве функций вида $h(z)=z^{2} u(z)$, где $u$ аналитична в диске $D=$ $\{z:\|z\|<\epsilon\}$ с нормой $\|h\|=\sup _{D}|u|$. Соответствующая функция $F$ аналитична при малых $z$ и является отображением сопряжения между (4.1) и его аппроксимацией при малых $y$.

Вопрос теперь в том, как определить особые точки функции $F$ в комплексной плоскости. Внутрь единичного диска функцию $F$ можно по индукции аналитически продолжить с помошью (4.3) следуюшим образом. Если $F$ аналитична в диске радиуса $r<1$, то (4.3) дает аналитическое продолжение на диск радиуса $\sqrt{r}$, если $F\left(z^{2}\right)$ не равна нулю или $4 / a$. Поскольку в диске радиуса $r$ функция $F$ предполагается аналитической, $F\left(z^{2}\right)$ не может обращаться в нуль, в противном случае, согласно (4.2), она обращалась бы в нуль бесконечно часто в окрестности нуля, что противоречит ее аналитичности при $z=0$ и $F^{\prime}(0)=-a$. Однако уравнение $F\left(z^{2}\right)=4 / a$ имеет решения на единичном диске, если $|a|>5$. Действительно, при $|a|>5$ можно легко показать, что если взять $F\left(z^{2}\right)=4 / a$, то итерация $(4.2)$ дает $F\left(z^{2^{n}}\right):=\epsilon_{n} \rightarrow 0$ при $n \rightarrow \infty$. Поскольку в силу условия $F^{\prime}(0)=-a$ функция $F$ является биекцией в окрестности $z=0$, при достаточно больших $n$ уравнение $F(z)=\epsilon_{n}$ имеет (единственное) решение $z=z_{0}$. Теперь, если $z_{1}$ таково, что $z_{1}^{2^{n}}=z_{0}$, имеем $F\left(z_{0}\right)=4 / a$. Однако тогда $F$ имеет точку ветвления типа квадратного корня при $z_{1}$, поскольку $F^{\prime}(z)$ не может обращаться в нуль нигде внутри единичного диска, иначе согласно (4.2) она бесконечно часто обрашалась бы в нуль вблизи начала координат.

Таким образом, при $|a|>5$ не имеется функций $F$ с только изолированными особыми точками. Действительно, как мы увидим ниже, в этом случае единичньй диск является барьером сингулярностей функции $F$.

Теперь рассмотрим случай, когда $|a|<5$. 
Мы утверждаем, что за исключением случая $a=-2$ точка $z=1$ является особой точкой функции $F$. Действительно, разложение функции в ряд Тейлора $F=\sum_{k=0}^{\infty} c_{k} \times$ $(z-1)^{k}$ дает $c_{0}=0$ или $c_{0}=a /(a-1)$. Непосредственно видно, что $c_{0}=0$ влечет $c_{k}=0$ при всех $k$, что противоречит условию $F^{\prime}(0)=-a$. Тогда $c_{0}=a /(a-1)$. Прямое вычисление показывает, что за исключением случая $a=-2\left(2^{k}-1\right)$ для некоторых $k \in \mathbb{Z}$ все $c_{k}$ равны нулю при $k \geqslant 1$, в противоречии с $F(0)=0$. Если $k>1$, то $|a|>5$. Тогда $k=1$, откуда следует $a=-2$. При таком $а$ уравнение (4.2) имеет явное решение

$$
F(z)=\frac{2 z}{z^{2}+z+1}
$$

Остается рассмотреть случай, когда $z=1$ является особой точкой функции $F$. Пусть $z=1$ является полюсом $F$. В этом случае из (4.2) получаем

$$
\lim _{z \rightarrow 1} \frac{a F\left(z^{2}\right)}{F(z)}=1 .
$$

Если $F(z)=\sum_{k=-p}^{\infty} c_{k}(z-1)^{k}-$ ряд Лорана функции $F$ при $z=1$, из уравнения (4.6) следует

$$
a=2^{p}
$$

откуда, поскольку $|a|<5$, имеем $a \in\{2,4\}$. При $a=2$ уравнение (4.2) имеет явное решение

$$
F(z)=\frac{2 z}{z-1}
$$

а при $a=4$ решением уравнения (4.2) является

$$
F(z)=-\frac{4 z}{(z-1)^{2}}
$$

Если функция $F$ не является мероморфной при $z=1$, то ее нельзя аналитически продолжить за единичную окружность.

Предположим сначала, что $F$ аналитична в открытом единичном диске $D_{1}$. Согласно уравнению (4.2), имеем

$$
F\left(z^{2^{n}}\right)=R_{n}(F(z))
$$

где $R_{n}$ - рациональная функция. Таким образом, если $z_{0}^{2^{n}}=1$ и $F$ аналитична при $z_{0}$, то она мероморфна при $z=1$, тогда как множество точек таких, что $z_{0}^{2^{n}}=1$, плотно на единичной окружности.

Пусть теперь $R<1$ - (максимальный) радиус аналитичности функции $F$. Уравнение (4.3) показывает, что на диске радиуса $R$ особые точки функции $F$ являются точками ветвления типа квадратного корня. С другой стороны, если $z_{0} \in D_{1}$ является алгебраической точкой ветвления и $z_{1}^{2}=z_{0}$, то $z_{1}$ также является алгебраической точкой ветвления, как ясно видно при продолжении уравнения (4.2) за небольшую окружность вблизи точки ветвления $z^{2}=z_{0}$. Тогда нетрудно видеть, что если $R<1$, то точки ветвления плотно накапливаются по направлению к единичной окружности. 
Наконец, функция $Q$, очевидно, аналитична в нуле. Второе свойство $Q$ можно показать, как в [1]. Действительно, из уравнения (4.2) следует, что $Q^{(2)}(s)=Q(R(s))$, где $R(s)=s^{2} /(a s-a)$. Если $s_{0} \in \partial \mathcal{K}_{p}$ является периодической точкой $R$, то либо $Q_{0}=0$ для любой точки периодической орбиты $s_{0}$, либо локальное разложение Тейлора показывает, что $Q$ не аналитична в любой точке орбиты $s_{0}$. Из сказанного следует, что $\partial \mathcal{K}_{p}$ - барьер сингулярностей функции $Q$.

\section{Список литературы}

[1] O. Costin ${ }^{1)}$, M. D. Kruskal. Equivalent of the Painlevé property for certain classes of difference equations and study of their solvability (to appear).

[2] B. L. J. Braaksma. Ann. Inst. Fourier. 1992. V. 42. № 3. P. 517-540.

[3] B. L. J. Braaksma. Transseries for a class of nonlinear difference equations. J. Diff. Equat. (to appear).

[4] O. Costin. Int. Math. Res. Notices. 1995. № 8. P. 377-417; Duke Math. J. 1998. V. 93. № 2. P. 289-344; O. Costin, M. D. Kruskal. Proc. Roy. Soc. London A. 1999. V. 455. P. 1931-1956; 1996. V. 452. № 1948. P. 1057-1085; O. Costin. Commun. Pure Appl. Math. 1999. V. 52. P. 0461-0478.

[5] O. Costin, R. D. Costin. Invent. Math. 2001. V. 145. № 3. P. 425-485.

[6] O. Costin, S. Tanveer. Commun. Pure Appl. Math. 2000. V. 53. P. 1092-1117; Existence and uniqueness of solutions of nonlinear evolution systems of $n$ th-order partial differential equations in the complex plane. (submitted); J. Écalle. Finitude des cycles limites et accéléro-sommation de l'application de retour. Preprint 90-36. Paris: Universite de Paris-Sud, 1990; Publ. Math. Orsay. 1981. № 81-05; M. D. Kruskal, H. Segur. Stud. Appl. Math. 1991. V. 85. № 2 . P. 129-181; Asymptotics Beyond All Orders. Eds. H. Segur, S. Tanveer, H. Levine. New York: Plenum, 1991.

[7] M. D. Kruskal, P. A. Clarkson. Stud. Appl. Math. 1992. V. 86. № 2. P. 87-165.

[8] N. Joshi. Irregular singular behavior in the first discrete Painlevé equation. In: SIDE III-Symmetries and Integrability of Difference Equations. CRM Proc. Lecture Notes. V. 25. Eds. D. Levi, O. Ragnisco. Providence, RI: AMS, 2000. P. 237-243.

[9] M. J. Ablowitz, R. Halburd, B. Herbst. Nonlinearity. 2000. V. 13. P. 889-905.

[10] B. Grammaticos, A. Ramani. Discrete Painlevé equations: derivation and properties. In: Applications of Analytic and Geometric Methods to Nonlinear Differential Equations. NATO ASI. Ser. C. V. 413. Ed. P. A. Clarkson. Dordrecht: Kluwer, 1993. P. 299-313.

[11] R. Conte, M. Musette. Rules of discretization for Painlevé equations. In: Theory of Nonlinear Special Functions: The Pailevé Transcendents. Eds. L. Vinet and P. Winternitz. Berlin: Springer, 1998.

[12] A. F. Beardon. Iteration of Rational Functions. New York: Springer, 1991.

\footnotetext{
${ }^{1)}$ См. статьи О. Костина (и др.) на http://www.math.rutgers.edu/ ${ }^{2}$ costin/paper_online.html
} 